\title{
Tailoring of Minimum Sidelobe Cosine-Sum Windows for High-Resolution Measurements
}

\author{
Hans-Helge Albrecht*
}

Physikalisch-Technische Bundesanstalt (PTB), Berlin, Germany

\begin{abstract}
Cosine-sum windows with minimum sidelobes (minimum sidelobe windows) have good properties in terms of peak sidelobe level (PSL) and equivalent noise bandwidth (ENBW). But neighboring windows (the number of coefficients differ by one) have quite large PSL differences. If, for a special data analysis, the PSL of the window should not exceed a given value, then often windows with a much lower PSL than specified have to be used. Due to increasing ENBW in the case of decreasing PSL, this leads, amongst others, to more uncertainty in the determination of signal amplitudes.

This article describes how to design modified minimum sidelobe windows which have similar properties to minimum sidelobe windows for a given PSL. Their ENBW were, however, traded off against PSL. Using such a design, windows can be created exactly for a given value of PSL at small ENBW. The adjustment of the asymptotic decay of the sidelobes and the determination of the window coefficients will be done without solving linear systems of equations to avoid known numerical problems. By using the proposed algorithm, more than 6000 windows with PSL values greater than $-350 \mathrm{~dB}$ were created. The parameters and coefficients of selected windows will be given in the article.
\end{abstract}

Keywords: Window function, spectral analysis, Fourier transform, signal processing.

\section{INTRODUCTION}

For the purpose of spectral analysis, often a short section of the duration $T_{w}$ is to be cut from a time varying signal. Spectral leakage occurs, if $T_{w}$ is not an integer multiple of the signal period. In the worst case, spectral leakage makes it impossible to detect neighboring spectral terms of small amplitude. This is a major problem in the spectral analysis of signals, when their period changes or is not known.

Window functions may reduce the effect of spectral leakage [1-3]. Due to their high peak sidelobe level, rectangular windows often cannot be used to detect weak signals in spectra with high dynamics. Windows with a low peak sidelobe level enable the detection of such signals, but they have a higher equivalent noise bandwidth than the rectangular window. This, amongst others, leads to larger uncertainties in the determination of the signal amplitudes.

Cosine-sum windows with minimum sidelobes (called minimum sidelobe windows in the following) [2, 4] have good properties in terms of both equivalent noise bandwidth and peak sidelobe level. Their weighting factors (as for all cosine-sum windows) can be determined very easily for different window lengths. For each number of window coefficients $C \geq 2$ with a given asymptotic decay of sidelobes of $20 \cdot(2 k+1) \mathrm{dB} /$ decade, with integer $k$ and $0 \leq k \leq(C-2)$, there is exactly one minimum sidelobe window. For peak sidelobe levels between $-43 \mathrm{~dB}$ and $-350 \mathrm{~dB}$ and a decay of the sidelobes of $20 \mathrm{~dB} /$ decade,

*Address correspondence to this author at the Physikalisch-Technische Bundesanstalt (PTB), Abbestraße 2-12, 10587 Berlin, Germany; Tel: +49 30 3481 7311; Fax: +49 303481 7490; E-mail: hans-helge.albrecht@ptb.de there are only 12 minimum sidelobe windows (parameters of the first 10 windows are given in [5]). The difference between values of neighboring windows for the equivalent noise bandwidth and the peak sidelobe level is quite large. In practice, for a given maximum peak sidelobe level, one should use the window with the highest permissible peak sidelobe level, and one has to accept in most cases a higher equivalent noise bandwidth than expected for a window designed with the given maximum peak sidelobe level.

The peak sidelobe level of cosine-sum windows can be traded off against the mainlobe width [2]. Based on this property, this article describes a method for modifying minimum sidelobe windows. Windows are presented which have similar low equivalent noise bandwidth to minimum sidelobe windows, but can be constructed exactly for a specified peak sidelobe level. They fill, therefore, the gap between neighboring minimum sidelobe windows. The design of modified minimum sidelobe windows for an asymptotic decay of the sidelobes $\geq 20 \mathrm{~dB} /$ decade is described. The adjustment of the decay of the sidelobes and the determination of the window coefficients will be performed without solving linear systems of equations to avoid known numerical problems. For six selected windows, the window parameters and coefficients will be given explicitly in Tables $\mathbf{1}$ and $\mathbf{2}$.

\section{THE COSINE-SUM WINDOW}

In this paragraph, the necessary theoretical background of the cosine-sum window function and its Fourier transform, of the asymptotic decay of sidelobes, of the shift of zeros of the Fourier transformed window function, of the determination of the coefficients by using the zeros, and of the determination of window parameters, is given. 
Due to the absence of all minus signs in [5], important equations are presented in the following subsection in a correct version.

\subsection{Window Function and its Fourier Transform}

The weighting function $w(t)$ of the cosine-sum window with real coefficients $A_{p}$ are as follows:

$w(t)=\left\{\begin{array}{cc}\sum_{p=0}^{G}(-1)^{p} A_{p} \cos \left(2 \pi p \frac{t}{T_{w}}\right), & \text { for } 0 \leq t \leq T_{w} \\ 0, & \text { otherwise }\end{array}\right.$

Its Fourier transform is the spectral window $W(f)$.

$W(f)=\frac{1}{T_{w}} \int_{0}^{T_{w}} w(t) e^{-j 2 \pi f t} \mathrm{~d} t$

Resulting from (1) and (2) with the normalized frequency ${ }^{1}$ $Q=f T_{w}$, one has

$W(Q)=\left(\frac{\sin (\pi Q)}{\pi} \sum_{p=0}^{G}(-1)^{p} A_{p} \frac{Q}{Q^{2}-p^{2}}\right) e^{-j \pi Q}$

with the limits

$\lim _{Q \rightarrow 0} W(Q)=A_{0}$

$\lim _{|Q| \rightarrow p} W(Q)=(-1)^{p} \frac{1}{2} A_{p}, \quad$ for $p=1,2, \ldots, G$

and

$|W(Q)|=\left|\frac{\sin (\pi Q)}{\pi} \sum_{p=0}^{G}(-1)^{p} A_{p} \frac{Q}{Q^{2}-p^{2}}\right|$

with the limits

$\lim _{Q \rightarrow 0}|W(Q)|=\left|A_{0}\right|$

$\lim _{|Q| \rightarrow p}|W(Q)|=\frac{1}{2}\left|A_{p}\right|, \quad$ for $p=1,2, \ldots, G$

Zeros of (3) and (5) occur with integer $Q$ for $|Q| \geq(G+1)$. Additional zeros produced by the term

$\sum_{p=0}^{G}(-1)^{p} A_{p} \frac{Q}{Q^{2}-p^{2}}$

can be used to optimize the window characteristics. Their location is determined by the coefficients $A_{p}$.

Term (7) can be written as the quotient of two polynomials. In the following, we consider the case of symmetric real zero pairs $\pm Q_{k}$. For one real zero pair, the following results

${ }^{1}$ In the following, the normalized frequency $Q$ is used. Bandwidths are also normalized and given without units.
$\left(Q+Q_{k}\right)\left(Q-Q_{k}\right)=Q^{2}-Q_{k}^{2}$

and with the constant $V_{1}$, the following is obtained for real coefficients $A_{p}$ and for the number of real zero pairs $G \geq 1$

$\sum_{p=0}^{G}(-1)^{p} A_{p} \frac{Q}{Q^{2}-p^{2}}=\frac{V_{1}}{Q} \prod_{k=0}^{G-1} \frac{Q^{2}-Q_{k}^{2}}{Q^{2}-(k+1)^{2}}$

Accordingly, (3) can be expressed in the form

$W(Q)=\left(\frac{\sin (\pi Q)}{\pi} \frac{V_{1}}{Q} \prod_{k=0}^{G-1} \frac{Q^{2}-Q_{k}^{2}}{Q^{2}-(k+1)^{2}}\right) e^{-j \pi Q}$

with the limits

$$
\begin{aligned}
& \lim _{Q \rightarrow 0} W(Q)=V_{1} \prod_{k=0}^{G-1} \frac{Q_{k}^{2}}{(k+1)^{2}} \\
& \lim _{|Q| \rightarrow p} W(Q)=V_{1} \frac{\prod_{k=0}^{G-1}\left(p^{2}-Q_{k}^{2}\right)}{2 \prod_{\substack{m=0 \\
m \neq p}}^{G}\left(p^{2}-m^{2}\right)} \text { for } p=1,2, \ldots, G
\end{aligned}
$$

\subsection{Adjustment of the Asymptotic Sidelobe Decay}

In the frequency domain, a different asymptotic decay of the sidelobes is reached by calculating the limits of the Fourier transformed window function for $L$ of the $G$ pairs of zeros $\left| \pm Q_{k}\right| \rightarrow \infty$. In the following, the case $L<G$ will be considered. Here at least one pair of zeros is available for the optimization of window properties.

With $V_{2}=\lim _{Q \rightarrow 0} W(Q)$ and (11a) and after formation of the limits $\left| \pm Q_{k}\right| \rightarrow \infty$ for $L$ of the whole of $G$ zero pairs, the following relation is obtained from (10)

$$
\begin{aligned}
W(Q)= & \left(\frac{\sin (\pi Q)}{\pi}(-1)^{L} \frac{V_{2}}{Q} \prod_{k=0}^{G-L-1} \frac{Q^{2}-Q_{k}^{2}}{Q_{k}^{2}}\right. \\
& \left.\cdot \prod_{k=1}^{G} \frac{k^{2}}{Q^{2}-k^{2}}\right) e^{-j \pi Q} \\
& \text { for } 0 \leq L<G \text { and } Q_{k} \neq 0
\end{aligned}
$$

with the limits

$\lim _{Q \rightarrow 0} W(Q)=V_{2}$

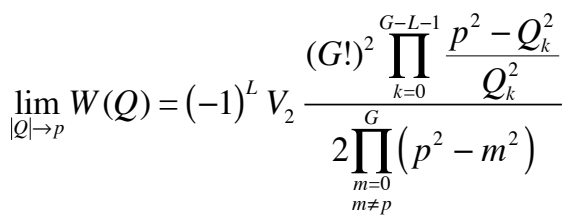

for $p=1,2, \ldots, G$

Some simplifications of (13b) give

$\lim _{|Q| \rightarrow p} W(Q)=(-1)^{p} V_{2} \prod_{k=0}^{p-1} \frac{G-k}{G+k+1} \prod_{k=0}^{G-L-1} \frac{Q_{k}^{2}-p^{2}}{Q_{k}^{2}}$

for $p=1,2, \ldots, G$ 
The gain of the spectral window is set using the constant $V_{2}$.

Calculating the limits $I \pm Q_{k} \mapsto \rightarrow$ of (12) and (14) for all other pairs of zeros, one gets the Fourier transformed RifeVincent window class I [6].

For $Q \gg G$, the sidelobes of (12) decay asymptotically with $Q^{-(2 L+1)}$, this means $20 \cdot(2 L+1) \mathrm{dB} /$ decade.

An approach in the time domain for the same asymptotic decay of the sidelobes, where the coefficients $A_{p}$ fulfill a linear system of equations derived from the derivatives of the window function $w(t)$, is given in [4].

\subsection{Shift of the Zeros $Q_{k}$}

Designing the cosine-sum window, it is of interest just how the shift of the pairs of zeros $\pm Q_{k}$ influences the Fourier transformed window function. With (12) one gets with a constant $Q$ for the differential

$$
\begin{gathered}
\mathrm{d} W\left(Q, Q_{k}\right)=2 \cdot W\left(Q, Q_{k}\right) \sum_{k=0}^{G-L-1} \frac{Q^{2}}{Q_{k}\left(Q_{k}^{2}-Q^{2}\right)} \mathrm{d} Q_{k} \\
\text { for } Q_{k}>0 \text { and }|Q| \neq Q_{k}
\end{gathered}
$$

Because for $Q_{k}>0$

$$
\frac{Q^{2}}{Q_{k}\left(Q_{k}^{2}-Q^{2}\right)} \begin{cases}>0, & \text { for }|Q|<Q_{k} \\ =0, & \text { for } Q=0 \\ <0, & \text { for }|Q|>Q_{k}\end{cases}
$$

the level of all sidelobes of $\left|W\left(Q, Q_{k}\right)\right|$ for $|Q|<Q_{k}$ will increase and for $|Q|>Q_{k}$ will decrease as a result of the shift of a zero $Q_{k}$ by $+\varepsilon$. With the increasing distance between the zero $Q_{k}$ and the current $\mathrm{Q}$, this influence from $\mathrm{d} Q_{k} / Q_{k}$ on $\mathrm{d} W\left(Q, Q_{k}\right)$ will decrease. This property allows a simple algorithm to minimize the sidelobe level. Thus, it is necessary to consider only the direct neighboring sidelobe maxima of a zero (see section 4 ).

\subsection{Calculation of the Window Coefficients $A_{p}$ from Zeros $Q_{k}$}

Using (3) and (12), a linear system of equations for the determination of the coefficients $A_{p}$ from the known zeros $Q_{k}$ can be set up. One degree of freedom remains to normalize the coefficients. Due to the limitations of computer arithmetic, it is difficult to obtain sufficient accuracy of the coefficients by solving the system of equations numerically.

However, the limits of the spectral window allow a direct determination of the coefficients $A_{p}$. This simple approach requires less numerical precision. From (4), (13a) and (14) the result for $Q_{k} \neq 0$ is

$$
\begin{aligned}
A_{0}= & V_{2} \\
A_{p}= & V_{2} \cdot 2 \prod_{k=0}^{p-1} \frac{G-k}{G+k+1} \prod_{k=0}^{G-L-1} \frac{Q_{k}^{2}-p^{2}}{Q_{k}^{2}} \\
& \text { for } p=1,2, \ldots, G \text { and } 0 \leq L<G
\end{aligned}
$$

The constant $V_{2}$ is used to normalize the coefficients. For the windows indicated in Tables $\mathbf{1}$ and $\mathbf{2}$, the coefficients fulfill the condition $\sum_{p=0}^{G} A_{p}=1$.

\subsection{Calculation of Window Parameters}

The mainlobe bandwidths and the peak sidelobe level can be obtained by using the window coefficients $A_{p}$ with (5) and (6). Also the peak signal gain and equivalent noise bandwidth can be calculated on the basis of the window coefficients.

\subsubsection{Peak Signal Gain}

The peak signal gain [2] is defined as $P S G=W(0)$. With (4a)

$P S G=A_{0}$

follows.

\subsubsection{Equivalent Noise Bandwidth}

The equivalent noise bandwidth of a window is the width of a rectangular window with the same peak power gain, which would accumulate the same noise power as the analyzed window [2]. The following applies for the equivalent noise bandwidth (normalized by $1 / T_{w}$ ) in the frequency range

$E N B W=\frac{\int_{-\infty}^{+\infty}|W(Q)|^{2} \mathrm{~d} Q}{W^{2}(0)}$

where $Q$ is the normalized frequency. Due to Parseval's theorem and if $w(t)=0$ for $t<0$ and $t>T_{w}$, (19) can be written as

$E N B W=\frac{\frac{1}{T_{w}} \int_{0}^{T_{w}} w^{2}(t) \mathrm{d} t}{W^{2}(0)}$

With (1) and (4a) one gets for the cosine-sum window with real coefficients $A_{p}$

$$
E N B W=\frac{1}{A_{0}^{2} T_{w}}\left[S+\sum_{p=0}^{G} A_{p}^{2} \int_{0}^{T_{w}} \cos ^{2}\left(2 \pi p \frac{t}{T_{w}}\right) \mathrm{d} t\right]
$$

with

$$
S=\sum_{i=0}^{G} \sum_{\substack{j=0 \\ j \neq i}}^{G}(-1)^{(i+j)} A_{i} A_{j}\left[\int_{0}^{T_{w}} \cos \left(2 \pi(i-j) \frac{t}{T_{w}}\right) \mathrm{d} t\right.
$$




$$
\left.+\int_{0}^{T_{w}} \cos \left(2 \pi(i+j) \frac{t}{T_{w}}\right) \mathrm{d} t\right]
$$

After integration (the definite integrals in (21b) are equal to $0)$ and simplification

$E N B W=\left\{\begin{array}{cc}1+\frac{1}{2 A_{0}^{2}} \sum_{p=1}^{G} A_{p}^{2}, & \text { for } G \geq 1 \\ 1, & \text { for } G=0\end{array}\right.$

is obtained. The equivalent noise bandwidth of the rectangular window is 1 . All cosine-sum windows with $G \geq 1$ have a higher equivalent noise bandwidth.

\section{DESIGN OF COSINE-SUM WINDOWS WITH ADJUSTABLE PEAK SIDELOBE LEVEL}

Using the design of minimum sidelobe windows with an asymptotic decay of sidelobes of $20 \cdot(2 L+1) \mathrm{dB} /$ decade based on the design for $L=0$ given in [5], the design of the modified minimum sidelobe windows will be described here for a given peak sidelobe level with a low equivalent noise bandwidth. These windows have similar properties to minimum sidelobe windows but the peak sidelobe level is traded off for equivalent noise bandwidth.

\subsection{Minimum Sidelobe Window}

To minimize the sidelobes all pairs of real zeros $\pm Q_{k}$ will be placed outside the mainlobe of the Fourier transformed window function. In this case the zero-crossing bandwidth of the main lobe is not given by one of the pairs of zeros, one gets $W(Q)$ for $|Q| \leq(G+1)$ the mainlobe and for $|Q|>(G+1)$ the sidelobes of the window function.

If the real zero pairs $\pm Q_{k}$ with $(G+1)<\left|Q_{0}\right| \leq\left|Q_{1}\right| \leq \ldots \leq\left|Q_{(G-L-1)}\right|$ are chosen so that for $|Q|>(G+1)$ the maximum of the function

$\left|W_{\text {norm }}(Q)\right|=\left|\frac{W(Q)}{W(0)}\right|$

becomes the minimum, the window is a minimum sidelobe window. Using (12) and (13a), (23) can be expressed as

$\left|W_{\text {norm }}(Q)\right|=\left|D_{1} D_{2} D_{3}\right|$

with

$D_{1}=\frac{\sin (\pi Q)}{\pi}$

$D_{2}=\frac{1}{Q} \prod_{k=0}^{G-L-1}\left(Q^{2}-Q_{k}^{2}\right) \prod_{k=1}^{G} \frac{1}{Q^{2}-k^{2}}$

$D_{3}=(G !)^{2} \prod_{k=0}^{G-L-1} \frac{1}{Q_{k}^{2}}$

Term $D_{2}$ in $(24 \mathrm{~b})$ allows the minimization of the sidelobes of the window function. Due to the symmetry of (24), only the case $Q \geq 0$ with positive zeros $+Q_{k}$ will be considered in the following. $D_{2}$ shows a local extremum between two neighboring positive zeros and for $Q>Q_{(G-L-1)}$ (Fig. 1). The multiplication of $D_{2}$ with $D_{1}$ forms - for $Q$ with $(G+1)<Q \leq Q_{0} \quad-$ at least one additional local extremum. $D_{3}$ is a constant for normalization only (Fig. 2).

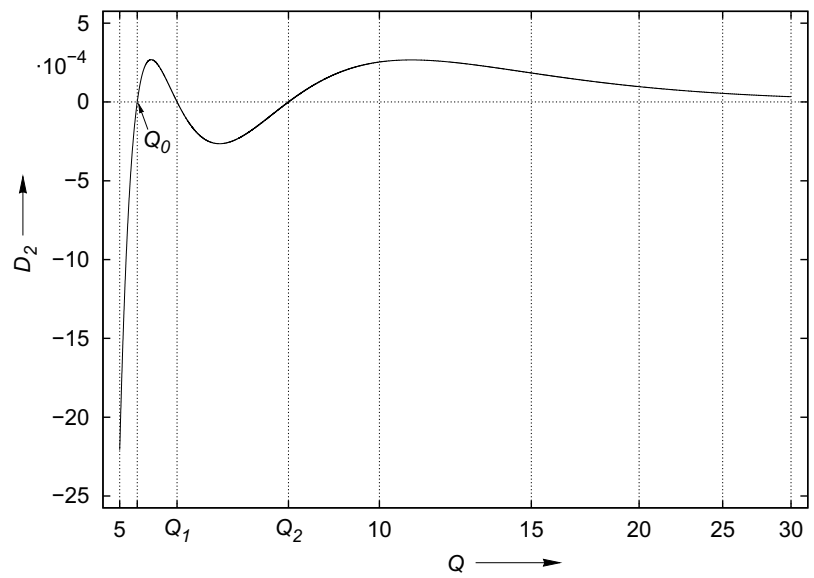

Fig. (1). Plot of $D_{2}(Q)$ for the 5-term minimum sidelobe window $(G=4, L=1)$.

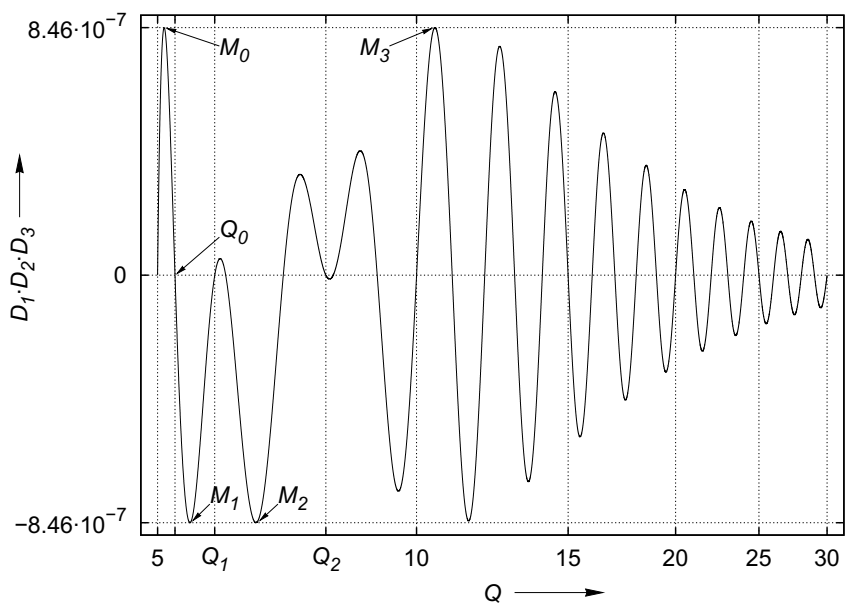

Fig. (2). Plot of $D_{1}(Q) \cdot D_{2}(Q) \cdot D_{3}$ for the 5-term minimum sidelobe window $(G=4, L=1) . M$ indicates the local maxima of the absolute value of the shown function.

Equation (24) has, therefore, within each of the intervals

$$
\begin{aligned}
& \left((G+1), Q_{0}\right],\left[Q_{0}, Q_{1}\right], \\
& \quad \ldots,\left[Q_{(G-L-2)}, Q_{(G-L-1)}\right],\left[Q_{(G-L-1)}, \infty\right)
\end{aligned}
$$

a local maximum $M_{k}$ for $k=0,1,2, \ldots, G-L$. When the zero $Q_{k}$ is shifted by the value $+\varepsilon$, the amplitude of the local maxima $M_{k}$ for $Q<Q_{k}$ increases and for $Q>Q_{k}$ decreases and vice versa (see subsection 2.3.).

In case of the same amplitude of all maxima, any shift of a zero by $\pm \varepsilon$ results in the increase of 
$\max \left(M_{0}, M_{1}, \ldots, M_{G-L}\right)$. It is, therefore, assumed that the minimal peak sidelobe level is reached here.

\subsection{Modified Minimum Sidelobe Window}

As well as the minimum sidelobe window also the modified minimum sidelobe window has pairs of zeros $\pm Q_{k}$. Due to the symmetry of the zeros in (24) only the case $Q \geq 0$ with positive zeros $+Q_{k}$ will be considered her.

Any shift of at least one zero of a minimum sidelobe window increases the peak sidelobe level. By modifying the window with the lower peak sidelobe level in such a way, one can adjust peak sidelobe levels between two neighboring minimum sidelobe windows (with equal $L$ ). In most cases, this procedure does not lead to windows with a low equivalent noise bandwidth for a given peak sidelobe level.

For the equivalent noise bandwidth of the cosine-sum window following (17) and (22) for $Q_{k} \neq 0$ one has

$$
E N B W=1+2 \sum_{p=1}^{G}\left(\prod_{k=0}^{p-1} \frac{G-k}{G+k+1} \cdot \prod_{k=0}^{G-L-1} \frac{Q_{k}^{2}-p^{2}}{Q_{k}^{2}}\right)^{2}
$$

for $G \geq 1$ and $0 \leq L<G$

If $Q_{k}>G$ for all $(G-L)$ zeros $Q_{k}$ of the Fourier transformed window function, then in (26) all differences will be $\left(Q_{k}^{2}-p^{2}\right)>0$. Each shift of $Q_{k}$ by the value $+\varepsilon$ leads to an increasing equivalent noise bandwidth.

In the following, the zero $Q_{0}$ will be used to control the peak sidelobe level. If, for a minimum sidelobe window with $(G-L) \geq 2$ the zero $Q_{0}$ with $Q_{0}>(G+1)$ will be shifted in the direction $Q=G$ with the remaining zeros unchanged, the equivalent noise bandwidth and the level of the maximum $M_{0}$ decreases. At the same time the level of all other maxima will increase. For $G \leq Q_{0} \leq(G+1)$ the maximum $M_{0}$ disappears and the zero-crossing bandwidth of the mainlobe will be determined by $Q_{0}$. For $Q_{0}=G$ the number of window coefficients decreases from $(G+1)$ to $G$, because the coefficient $A_{G}$ vanishes (17).

If $Q_{0}$ is fixed, $G-L-1$ zeros remain, which can be chosen in such a way that a low equivalent noise bandwidth is reached at a given peak sidelobe level. For a given peak sidelobe level lying between the values of two neighboring minimum sidelobe windows, it is possible to create windows with different equivalent noise bandwidth, but with equal $L$ and $G$. Simulations for $L=0$ and $G=2$, as well as $L=0$ and $L=1$ for $G=3$, show that the window with the same level of the sidelobe maxima $M_{1}$ up to $M_{G-L}$ has the lowest equivalent noise bandwidth. This condition is similar to that for minimum sidelobe windows. But in the case of modified minimum sidelobe windows, the sidelobe maximum $M_{0}$ (if it exists) is not considered, because it does not influence the peak sidelobe level.
The equivalent noise bandwidth and the peak sidelobe level are shown as a function of both zeros $Q_{0}$ and $Q_{1}$ for windows with $G=2$ and $L=0$ in Fig. (3) and for windows with $G=3$ and $L=1$ in Fig. (4), respectively. For a constant $Q_{0}$ with an increasing $Q_{1}$, starting with $Q_{1}=Q_{0}$, the equivalent noise bandwidth increases due to the increasing distance of the zero $Q_{1}$ from the mainlobe (26). At the same time, the level of the maximum $M_{1}$ increases and the level of the maximum $M_{2}$ and, consequently, the peak sidelobe level decreases. This behavior persists until the two maxima $M_{1}$ and $M_{2}$ neighboring the zero $Q_{1}$ have the same level (dots in Figs. 3 and 4). If $Q_{1}$ increases further, the equivalent noise bandwidth and the peak sidelobe level then determined by the level of $M_{1}$ increase.

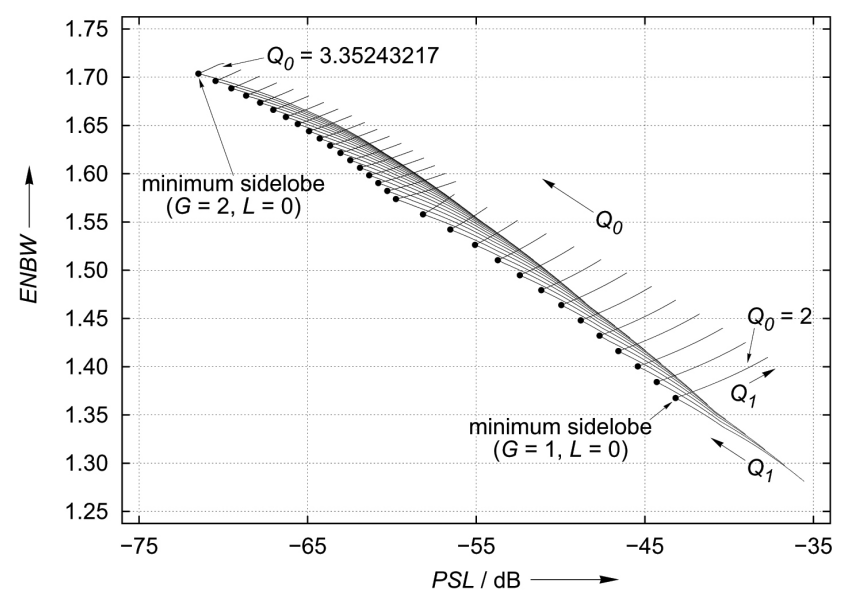

Fig. (3). Equivalent noise bandwidth $(E N B W)$ and peak sidelobe level (PSL) as a function of the zeros $Q_{0}$ and $Q_{1}$ of cosine-sum windows with $G=2$ and $L=0$. For $Q_{0}=2$, windows follow with $G=1$ and $L=0$.

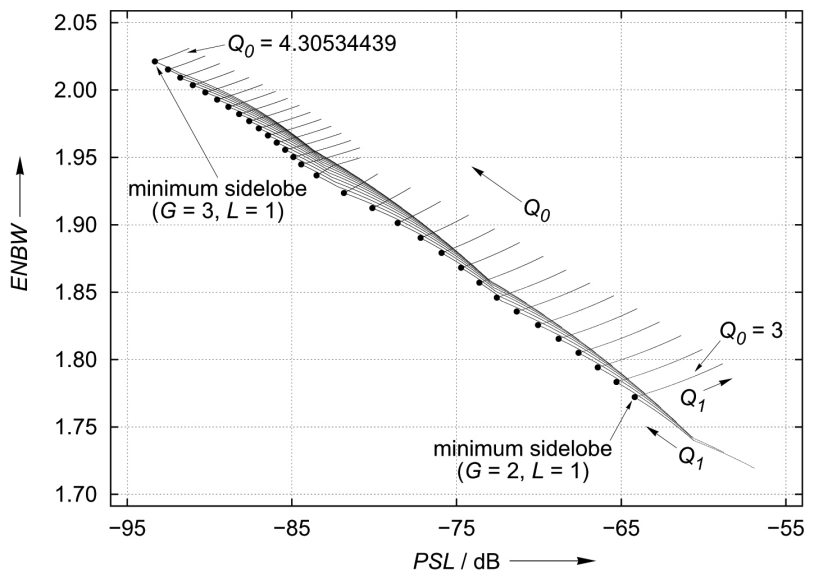

Fig. (4). The same as Fig. (3) but for cosine-sum windows with $G=3$ and $L=1$. For $Q_{0}=3$, windows follow with $G=2$ and $L=1$.

Fig. (5) shows $\left|W_{\text {norm }}(Q)\right|(24)$ for the minimum sidelobe window and for a modified minimum sidelobe window (both 
windows for $G=2$ and $L=0$ ), where the peak sidelobe level of the modified minimum sidelobe window is only about $2 \mathrm{~dB}$ lower and the equivalent noise bandwidth somewhat higher than for the minimum sidelobe window with $G=1$ and $L=0$. Note the lower mainlobe zerocrossing bandwidth of the modified window due to $Q_{0}$.

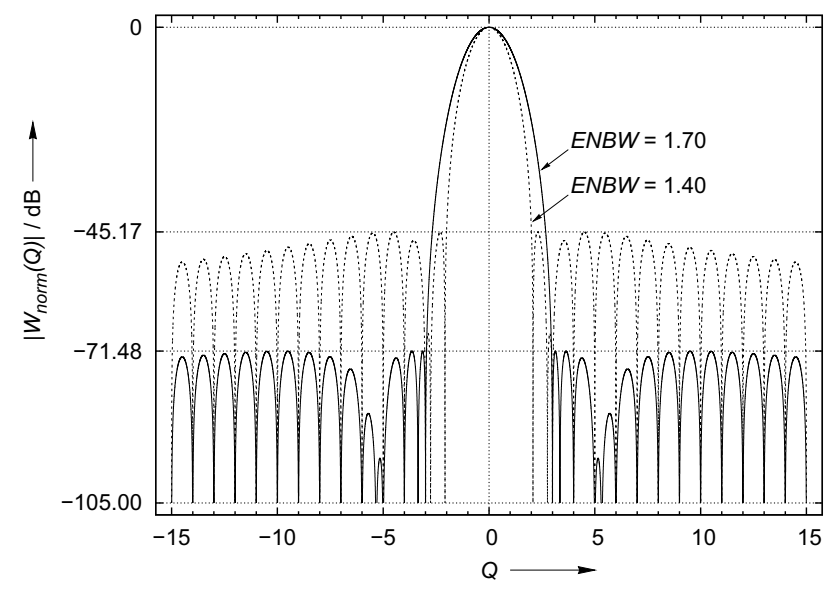

Fig. (5). Absolute value of the normalized Fourier transformed minimum sidelobe window (solid line) and of a modified minimum sidelobe window (dashed line), both windows with $G=2$ and $L=0$.

For the design of modified minimum sidelobe windows with a given peak sidelobe level it is possible to develop a simple algorithm on the basis of simulation results. Starting with a minimum sidelobe window with desired $L$ (where $(G-L) \geq 2$ ) and next lower peak sidelobe level, $Q_{0}$ will be shifted in the direction of $Q=G$. The remaining $G-L-1$ zeros have to be optimized in such a way, that the maxima $M_{1}$ up to $M_{G-L}$ of $\left|W_{\text {norm }}(Q)\right|$ (24) reach the same level. Because there is a non-linear relation between $Q_{0}$ and the peak sidelobe level, $Q_{0}$ has to be optimized so that the modified minimum sidelobe window has the desired peak sidelobe level.

$D_{1}(Q) \cdot D_{2}(Q) \cdot D_{3}$ of a modified 5-term minimum sidelobe window designed by the proposed algorithm is shown in Fig. (6) in comparison to the 5-term minimum sidelobe window (see also Fig. 2). The values for $|Q| \leq 5$ belonging to the mainlobe are not shown.

\section{DETERMINATION OF THE ZEROS}

\subsection{Zeros of the Minimum Sidelobe Window}

For the optimization of the zeros for the same level of the maxima, a simple algorithm is used. The result of the first step is an approximation of the zeros. In the next step, the zeros will be determined with high accuracy.

\subsubsection{First Step, Approximated Zeros}

The following initial values can be used for the zeros:

$Q_{k}=G+k+2$, for $k=0,1, \ldots,(G-L-1)$

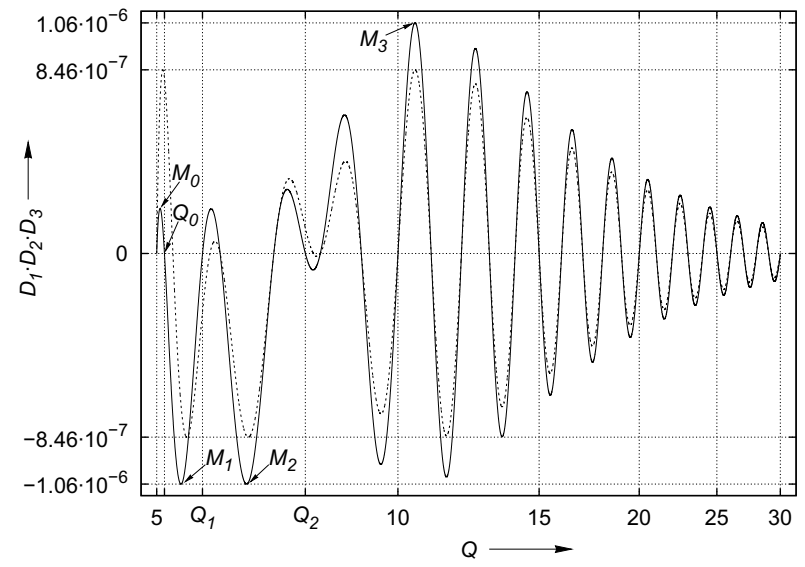

Fig. (6). Plot of $D_{1}(Q) \cdot D_{2}(Q) \cdot D_{3}$ for a modified 5-term minimum sidelobe window (solid line) and for comparison the 5-term minimum sidelobe window (dashed line), $G=4$ and $L=1$ for both windows. $M$ indicates the local maxima of the absolute value of the shown function.

At the beginning of each optimization cycle, the level $M_{k}$ and the position of the maxima $Q_{M_{k}}$ for $k=0,1, \ldots,(G-L)$ are determined by using (12). Additionally the ratio

$R=\frac{\max \left(M_{0}, M_{1}, \ldots, M_{G-L}\right)}{\min \left(M_{0}, M_{1}, \ldots, M_{G-L}\right)}$

is calculated. If $R$ falls below a given value, approximations for all zeros are found. Otherwise, for each zero a step width $\Delta Q_{k}$ will be calculated by using the distance between neighboring maxima with a fixed number of steps $S$ :

$\Delta Q_{k}=\frac{Q_{M_{k+1}}-Q_{M_{k}}}{S}$

Each zero $Q_{k}$ is shifted by $+\Delta Q_{k}$, i.e., $Q_{k}:=Q_{k}+\Delta Q_{k}$, if the levels of neighboring maxima $M_{k}<M_{k+1}$. The zero will be shifted by $-\Delta Q_{k}$, i.e., $Q_{k}:=Q_{k}-\Delta Q_{k}$, if $M_{k}>M_{k+1} \cdot Q_{k}$ is not changed in the case of $M_{k}=M_{k+1}$. In the next step, the optimization cycle starts again with the calculation of the level and the position of the maxima.

For $R=1.2$ and $S=100$, the zeros of windows with a peak sidelobe level $>-350 \mathrm{~dB}$ were approximated. If $S$ is chosen to be too small, it is not possible to obtain adequate small values of $R$. In the other case, only the number of optimization cycles will grow.

\subsubsection{Second Step, High Accuracy Zeros}

Initial values for the optimization are the approximated zeros and the associated $\Delta Q_{k}$. At the beginning of each optimization cycle, levels and positions of the maxima and $R$, as described, will be calculated. If $R$ is smaller than all previous values, $R$ and the related zeros will be stored. The zeros will be shifted in the same way as described for the approximation, and the optimization cycle starts again. If $Z$ consecutive optimization cycles do not result in a decrease of 
$R$, the values of all $\Delta Q_{k}$ will be divided by two. If during the shifting of a zero one gets $Q_{k} \pm \Delta Q_{k}=Q_{k}$ due to the limited accuracy of the arithmetic used, $\Delta Q_{k}$ will be doubled and then the zero $Q_{k}$ will be shifted. In all the following optimization cycles, $\Delta Q_{k}$ is not decreased. The optimization ends, if thus after $Z$ consecutive optimization cycles without decreasing of $R$, no $\Delta Q_{k}$ can be divided by two.

For zero optimization of the windows stated in Tables $\mathbf{1}$ and $2, Z=4$ was used.

\subsection{Zeros of the Modified Minimum Sidelobe Window}

In the case of modified minimum sidelobe windows only $G-L-1 \quad$ zeros $\quad Q_{k} \quad$ with $\quad k=1,2, \ldots,(G-L-1) \quad$ are available for minimizing the peak sidelobe level, because with the given zero $Q_{0}$ the achievable peak sidelobe level, or respectively the equivalent noise bandwidth was determined. $Q_{0}$ has to fulfill the condition $G<Q_{0}<Q_{0 / \mathrm{MSL}}$, where $Q_{0 / \mathrm{MSL}}$ is the zero $Q_{0}$ of the minimum sidelobe window with the same $G$ and $L$ as the modified minimum sidelobe window.

The minimization of the peak sidelobe level is performed with an algorithm which is similar to that described for the minimum sidelobe window. The zero $Q_{0}$ remains unchanged and the maximum $M_{0}$ (if it exists) will not be considered during the calculation of $R$. Further differences compared to the described algorithm are the initial values of the zeros

$Q_{k}=Q_{0}+k$, for $k=1,2, \ldots,(G-L-1)$

and the calculation of $R$

$R=\frac{\max \left(M_{1}, M_{2}, \ldots, M_{G-L}\right)}{\min \left(M_{1}, M_{2}, \ldots, M_{G-L}\right)}$

\section{RESULTS}

By using the described algorithm, the zeros and coefficients of cosine-sum windows with a peak sidelobe level of $>-350 \mathrm{~dB}$ and $L=0,1, \ldots, 21$ were calculated. In the case of sufficient numerical accuracy of the coefficients, $L=21$ corresponds to an asymptotic decay of the sidelobes of $860 \mathrm{~dB} / \mathrm{dec} a d e$. The difference of the peak sidelobe level of neighboring windows with equal $L$ is not greater than 0.5 dB. In Fig. (7) the equivalent noise bandwidth is shown as a function of the peak sidelobe level. Modified minimum sidelobe windows (small dots, appearing as a line in the first plot) have similar features to minimum sidelobe windows (large dots). The modified minimum sidelobe windows close the gap between minimum sidelobe windows with respect to equivalent noise bandwidth and peak sidelobe level.

Some of the minimum sidelobe windows shown in Fig. (7) are well known. In the case of rounded coefficients (2 decimal places), the 2-term window $(G=1, L=0)$ is identical to the Hamming window [1]. All 3- and 4-term windows $(G=2$, respectively $G=3, L=0,1, \ldots, G-1)$ show a very good agreement with the windows given in [4].
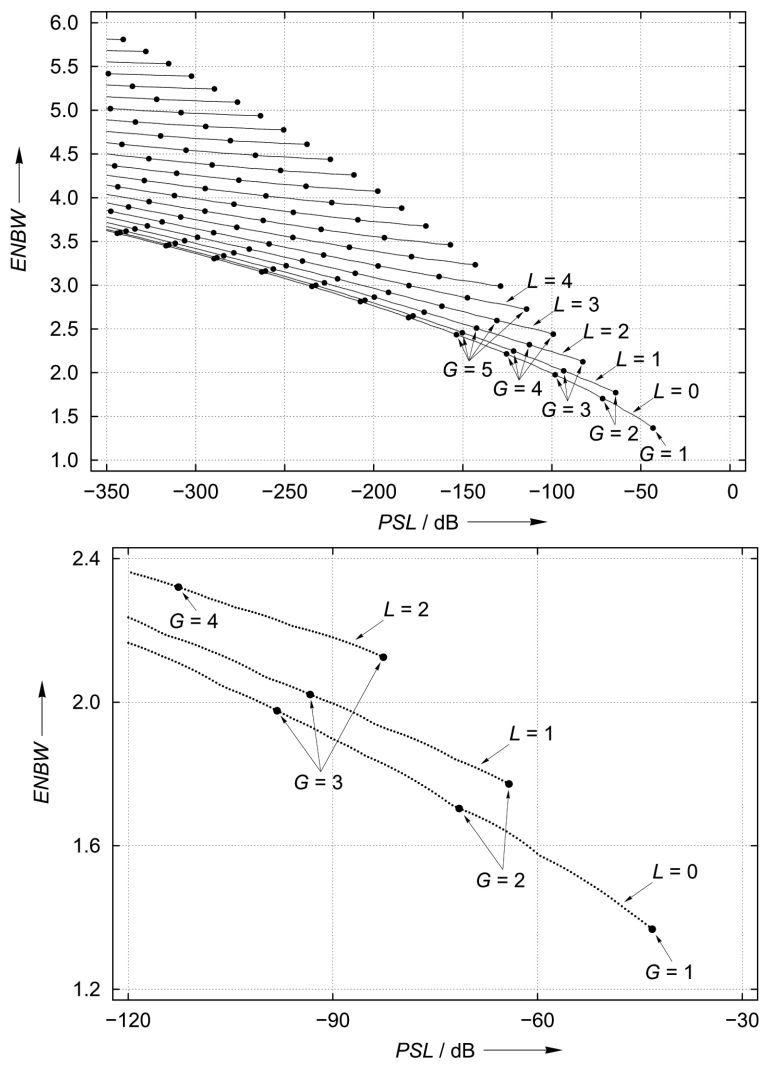

Fig. (7). Equivalent noise bandwidth $(E N B W)$ as a function of the peak sidelobe level (PSL) of modified minimum sidelobe windows designed with the described algorithm (small dots, appearing as a line in the first plot). In comparison minimum sidelobe windows (large dots).

The described window design algorithm was implemented using the $\mathrm{C}$ programming language. The lowest achievable peak sidelobe level of the window is limited by the accuracy of the computer arithmetic. The long double type in $\mathrm{C}$ is the floating point data type with the highest available accuracy. For the computer used it corresponds to the double extended precision floating point data format in [7] and allows the design of windows with a peak sidelobe level greater than $-350 \mathrm{~dB}$. Using this data format, the implementation of the algorithm is on the safe side, because nowadays and probably for the near future the required peak sidelobe level for high resolution measurements should be significant greater than $-350 \mathrm{~dB}$.

Equation (12) to calculate $W(Q)$ by using the zeros $\pm Q_{k}$ has good properties in terms of numerical requirements on the arithmetic accuracy. In the case of decaying sidelobe levels the difference of the values calculated for $W(Q)$ by using the coefficients $A_{p}$ and (3) differ increasingly from the values calculated with (12) (differences of almost the same numbers). If $W(Q)$ is calculated by using the zeros, it is possible to design windows with a peak sidelobe level far 
below $-350 \mathrm{~dB}$. In this case, it is no longer possible to reproduce $W(Q)$ by using the coefficients $A_{p}$. Independent of both equations, the spectrum of the window function can be determined by FFT and zero padding. The normalized amplitude spectrum of two modified minimum sidelobe windows determined in this way is shown in Fig. (8) (FFT of one data set with $2^{24}$ samples, window length 65587 samples). The peak sidelobe levels of $-85.06 \mathrm{~dB}$ and $-349.95 \mathrm{~dB}$ calculated by using the coefficients $A_{p}$ and (5) are in good agreement with the values determined from the spectrum.

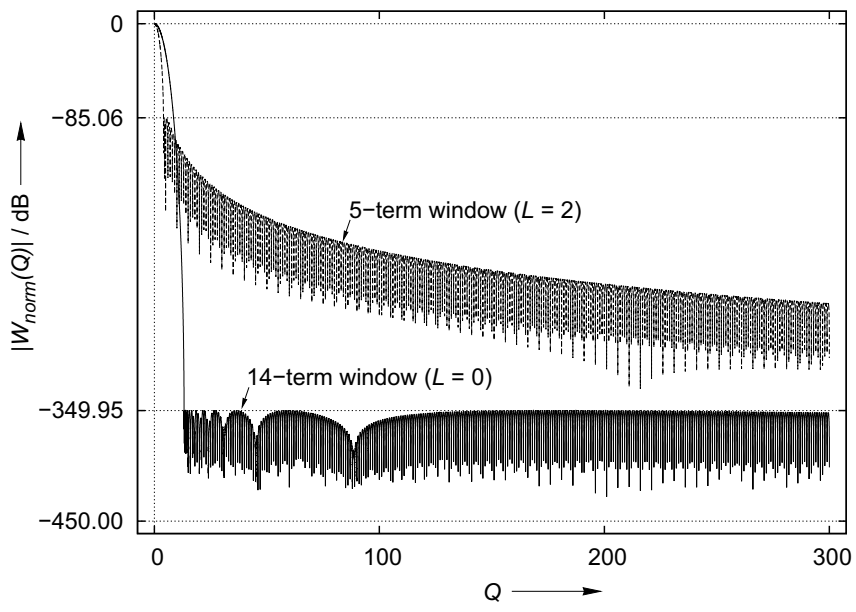

Fig. (8). FFT plot of a modified 5-term (broken line) and a modified 14-term minimum sidelobe window (solid line).

Parameters and coefficients of selected windows are given in Tables $\mathbf{1}$ and $\mathbf{2}$. The bandwidths and the scallop loss were calculated by using (5), the peak signal gain and the equivalent noise bandwidth by using (18) and (22). The values for the peak sidelobe level are rounded up. To reach a good approximation for the computer internal number representation, the window coefficients are given with more decimal places than necessary for the accuracy of the used arithmetic.

Windows 1 to 3 have an asymptotic decay of the sidelobes of $60 \mathrm{~dB} /$ decade and windows 4 to 6 of 140
$\mathrm{dB} /$ decade. The peak sidelobe level of the modified minimum sidelobe windows 2 and 5 is located between the values of the neighboring minimum sidelobe windows 1 and 3 , or respectively, 4 and 6 . For windows 2 and 5 the zero-crossing bandwidth of the mainlobe is $<2(G+1)$, meaning it is determined by the zero pair $\pm Q_{0}$. Plots of $\left|W_{\text {norm }}(Q)\right|$ of the windows 2 and 5 , calculated by using the coefficients $A_{p}$, are shown in Figs. (9 and 10).

A last example shows that for a given maximum sidelobe level, the use of modified minimum sidelobe windows enables a clear decrease of the width of the mainlobe and the equivalent noise bandwidth compared to what can be achieved by using a minimum sidelobe window. Part of the normalized power spectrum of the output signal of a simulated 8 bit ADC with additive noise at full-scale triggering with a sine signal and a sample frequency of $100 \mathrm{MHz}$ is represented in Fig. (11). The averaged power spectra $(N=100)$ were calculated on the basis of data sets with 32327 samples of the signal. The number of spectral lines was increased for the plot by zero padding (total length of the data set $2^{21}$ samples). The scale was adapted to ensure a constant signal level. In the given example the maxima of the sidelobes of windows used have to be at least $15 \mathrm{~dB}$ below the noise of the ADC output signal. The 5-term minimum sidelobe window [5] with $L=0$ and $E N B W=2.21535$ (dashed line) fulfills the requirement in contrast to the 4-term minimum sidelobe window, but it has a peak sidelobe level less than required and, consequently, a broadened mainlobe and an increased equivalent noise bandwidth compared to the modified 5-term minimum sidelobe window with $L=0$ and $E N B W=2.00415$ (solid line). The spectra of the sampled but not quantized ADC sine wave input signal (dotted lines) show that the sidelobes of the modified window are $104.2 \mathrm{~dB}$ below the power of the driving sine wave signal $P_{\text {sine }}$ and about $15.7 \mathrm{~dB}$ below the noise of the ADC output signal, near the required value of $15 \mathrm{~dB}$. In contrast, for the 5-term minimum sidelobe window the peak sidelobe level is about $40.3 \mathrm{~dB}$ below the noise.

Table 1. Parameters of Selected Cosine-Sum Windows

\begin{tabular}{|c|c|c|c|c|c|c|c|c|c|}
\hline Window & L & G & $\begin{array}{c}\text { Peak Sidelobe } \\
\text { Level in dB }\end{array}$ & ENBW & $\begin{array}{c}\text { Peak Signal } \\
\text { Gain in dB }\end{array}$ & $\begin{array}{c}\text { Scallop Loss } \\
\text { in dB }\end{array}$ & $\begin{array}{c}\text { 3.0-dB } \\
\text { Bandwidth }\end{array}$ & $\begin{array}{c}\text { 6.0-dB } \\
\text { Bandwidth }\end{array}$ & $\begin{array}{c}\text { Zero Crossing } \\
\text { Bandwidth }\end{array}$ \\
\hline \hline 1 & 1 & 8 & -232.523 & 2.99869 & -12.48929 & 0.37454 & 2.82255 & 3.97931 & 18.00000 \\
\hline 2 & 1 & 9 & -246.429 & 3.08023 & -12.72558 & 0.35519 & 2.89874 & 4.08740 & 19.01607 \\
\hline 3 & 1 & 9 & -260.832 & 3.16222 & -12.95664 & 0.33721 & 2.97537 & 4.19608 & 20.00000 \\
\hline 4 & 3 & 8 & -220.335 & 3.07249 & -12.69763 & 0.35654 & 2.89251 & 4.07736 & 18.00000 \\
\hline 5 & 3 & 9 & -234.774 & 3.14900 & -12.91506 & 0.33968 & 2.96386 & 4.17875 & 19.03439 \\
\hline & 3 & 9 & -249.214 & 3.22289 & -13.11970 & 0.32449 & 3.03282 & 4.27667 & 20.00000 \\
\hline
\end{tabular}


Table 2. Coefficients of Selected Cosine-Sum Windows

\begin{tabular}{|c|c|c|c|}
\hline$A_{0}$ & $2.374298741532465928226 \cdot 10^{-01}$ & $2.310581202331358499435 \cdot 10^{-01}$ & $2.249924617087535177329 \cdot 10^{-01}$ \\
\hline$A_{1}$ & $3.994704373801009358001 \cdot 10^{-01}$ & $3.922514736021656858831 \cdot 10^{-01}$ & $3.851495428292902693259 \cdot 10^{-01}$ \\
\hline$A_{2}$ & $2.362644608100282475133 \cdot 10^{-01}$ & $2.385553629158978655597 \cdot 10^{-01}$ & $2.403597686865028390968 \cdot 10^{-01}$ \\
\hline$A_{3}$ & $9.620676838363516649024 \cdot 10^{-02}$ & $1.021288669149117706979 \cdot 10^{-01}$ & $1.077408077837851454781 \cdot 10^{-01}$ \\
\hline$A_{4}$ & $2.591512168016078991738 \cdot 10^{-02}$ & $2.977294169292394185833 \cdot 10^{-02}$ & $3.373630665800276621290 \cdot 10^{-02}$ \\
\hline$A_{5}$ & $4.307708101213669512442 \cdot 10^{-03}$ & $5.586700597441296634013 \cdot 10^{-03}$ & $7.046059650969717333158 \cdot 10^{-03}$ \\
\hline$A_{8}$ & $1.320024271202038321705 \cdot 10^{-07}$ & $5.899889578740096042846 \cdot 10^{-07}$ & $1.853811776589548714026 \cdot 10^{-06}$ \\
\hline$A_{9}$ & & $9.538390427238738941684 \cdot 10^{-10}$ & $1.152831741603563323368 \cdot 10^{-08}$ \\
\hline
\end{tabular}

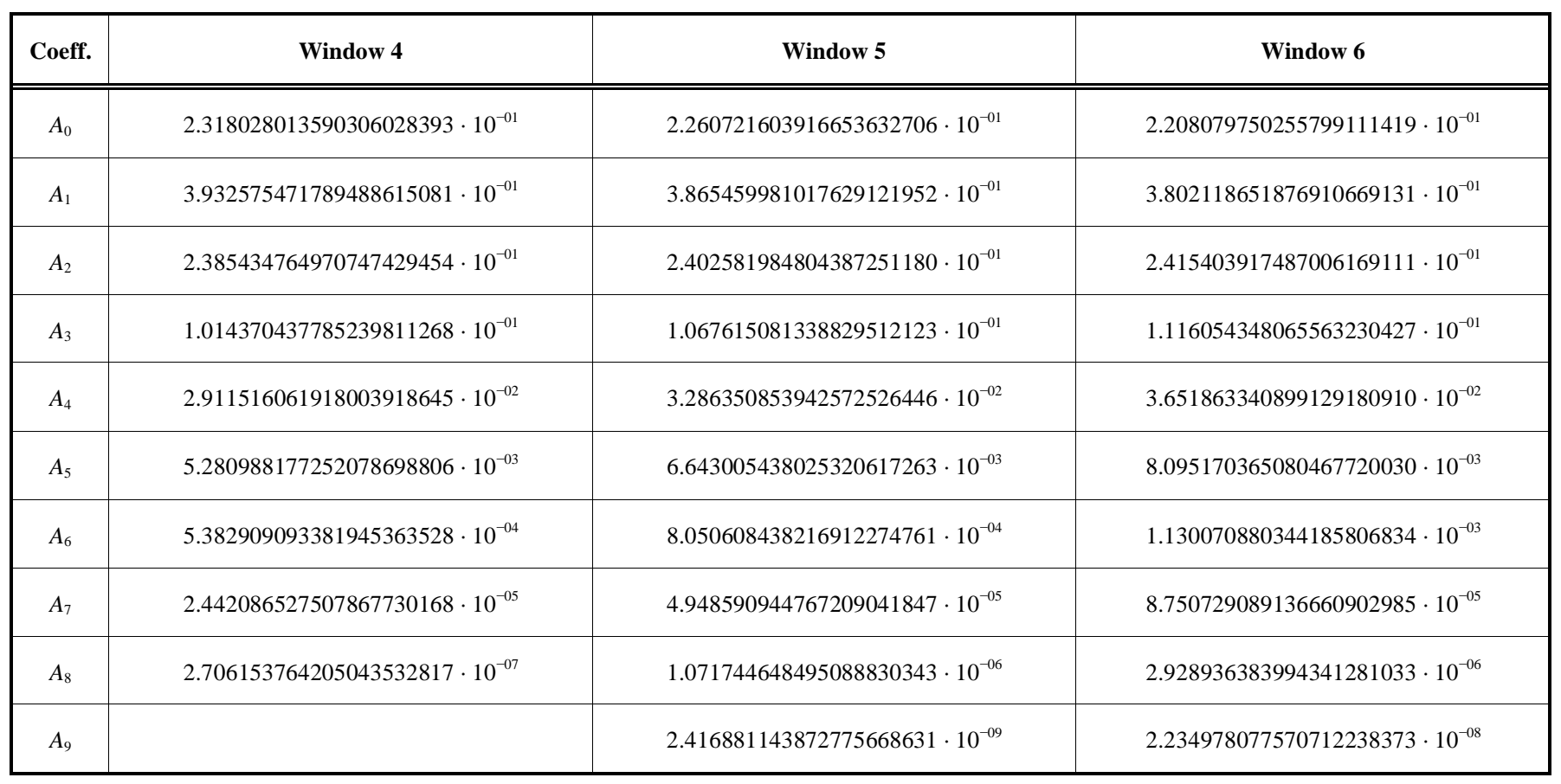

\section{SUMMARY}

The modified minimum sidelobe windows presented here have similar properties to conventional minimum sidelobe windows. In contrast to conventional minimum sidelobe windows, modified minimum sidelobe windows can be designed for a given peak sidelobe level by continuously trading the peak sidelobe level for the equivalent noise bandwidth. This enables the adaptation of these window parameters to the processed data. Based on the zeros of the
Fourier transformed window function the design algorithms for both windows are given. By using the described algorithms the coefficients of window functions with a peak sidelobe level higher than $-350 \mathrm{~dB}$ and different asymptotic decay of the sidelobes were determined without solving a system of linear equations. The parameters and coefficients of selected window functions are presented. A catalog with coefficients and parameters of more than 6000 minimum sidelobe windows, or respectively, modified minimum sidelobe windows, is in preparation. 


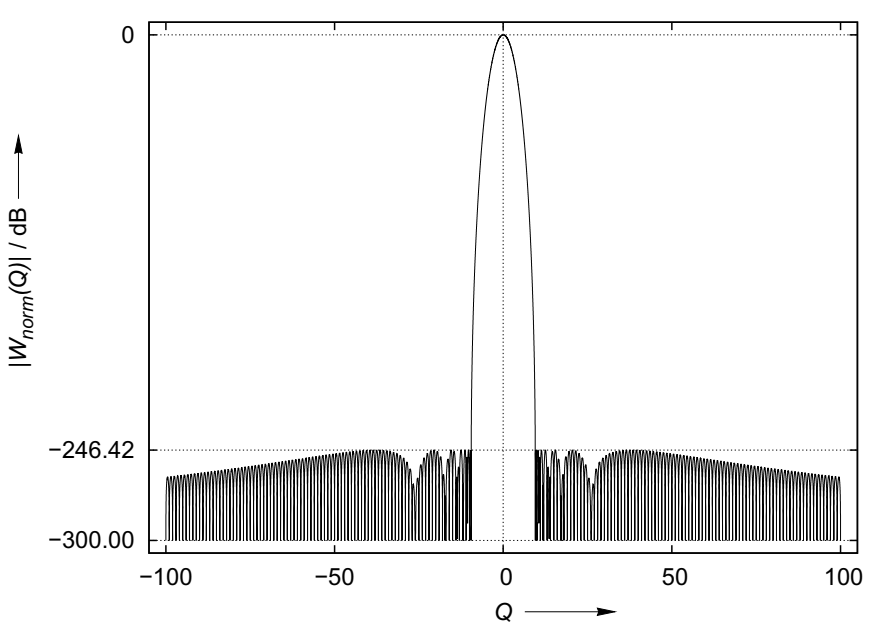

Fig. (9). Plot of $\left|W_{\text {norm }}(Q)\right|$ of the modified minimum sidelobe window 2 (see Tables $\mathbf{1}$ and $\mathbf{2}$ ).

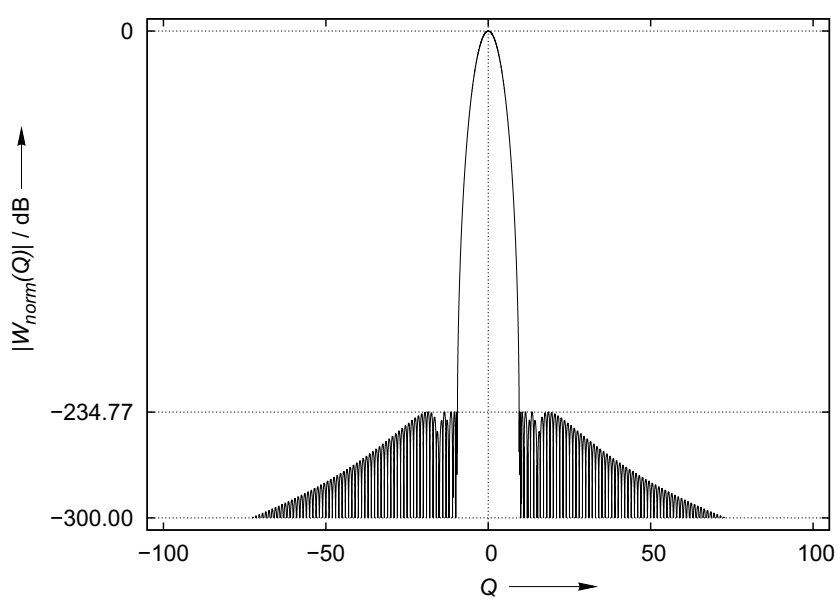

Fig. (10). Plot of $\left|W_{\text {norm }}(Q)\right|$ of the modified minimum sidelobe window 5 (see Tables $\mathbf{1}$ and $\mathbf{2}$ ).

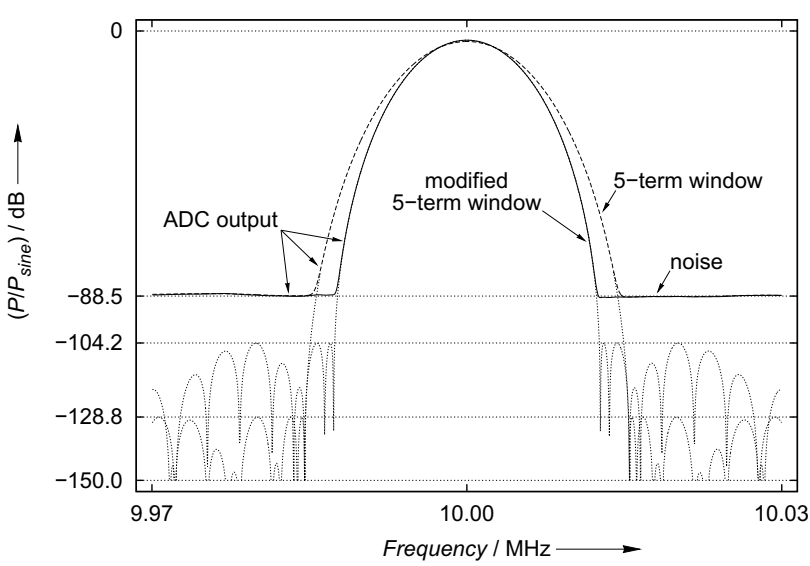

Fig. (11). Normalized power spectrum of the output signal of a simulated ADC (sine wave input) using different windows. Hidden spectra of the windows (dotted lines).

\section{ACKNOWLEDGEMENT}

The author would like to thank Dr. Alfred Link for his fruitful discussions.

\section{REFERENCES}

[1] R. B. Blackman and J. W. Tukey, The Measurement of Power Spectra, New York: Dover Publications Inc., 1958

[2] F. J. Harris. "On the use of windows for harmonic analysis with discrete fourier transform," Proc. IEEE, vol. 66. no. 1, pp. 51-83, January 1978.

[3] O. M. Solomon, Jr., "The use of DFT windows in signal-to-noise ratio and harmonic distortion computations," IEEE Trans. Instrum. Meas., vol. 43, pp. 194-199, April 1994.

[4] A. H. Nuttall. "Some windows with very good sidelobe behavior," IEEE Trans. Acoust., Speech, Signal Process., vol. ASSP-29, no. 1, pp. 84-91, February 1981.

[5] H.-H. Albrecht, "A family of cosine-sum windows for highresolution measurements," Proc. IEEE Int. Conf. Acoust., Speech, Signal Process., vol. 5, Salt Lake City, Utah, May 2001, pp. 30813084.

[6] D. C. Rife and G. A. Vincent, "Use of the discrete fourier transform in the measurement of frequencies and levels of tones," Bell Syst. Tech. J., vol. 49, no. 2, pp. 197-228, February 1970.

[7] Intel ${ }^{\circledR} 64$ and IA-32 Architectures Software Developer's Manual. Intel Corporation, November 2006.

(C) Hans-Helge Albrecht; Licensee Bentham Open.

This is an open access article licensed under the terms of the Creative Commons Attribution Non-Commercial License (http://creativecommons.org/licenses/by-nc/3.0/) which permits unrestricted, non-commercial use, distribution and reproduction in any medium, provided the work is properly cited. 